\title{
Public Health Decision-Making in the Real World: Four Points to Reshape It After COVID-19
}

\author{
Emanuele Torri, MD (1); Giandomenico Nollo, PhD
}

Key Words: public health practice, public health, quality of health care, risk management, safety management

$\mathrm{T}$ he coronavirus disease 2019 (COVID-19) outbreak is rapidly progressing worldwide, tremendously impacting health systems, science, and society. ${ }^{1}$ Uncertainty is unparalleled in living memory, and involves the urgency of deciding with limited resources and under pressures from conflicting interests. Drawing from the hard lessons of Italy's experience, the European country most affected by the pandemics to date, ${ }^{2}$ we wish to share ideas to improve emergency decision-making.

\section{GOVERNANCE}

Expanding international coordination ${ }^{3}$ and healthcare accreditation programs to guarantee reliability of hygiene practices, surveillance, and safety systems on the ground is crucial now. A special attention toward strengthening education for leadership development of public health officials and clinicians is needed to enable engagement, resilience, and trust.

\section{DATA-DRIVEN MANAGEMENT}

Information and data sets for COVID-19 research should be in the public domain, to provide broader development of models and data supporting health authorities and agencies in public decisions. Peer observations and bottom-up inputs should be collected. Multiple scientific competence centers should be institutionalized as "scientific civil protection" for activations during emergencies to perform timely analysis and experiments tailored on policy questions.

\section{HEALTH TECHNOLOGY ASSESSMENT}

Context-based analysis of cultural, organizational, economic, social, and ethical issues is vital given the complexity of public health. First, politicians and the public should be thoroughly educated in health technology assessment. Second, we need practical models to expedite the appraisal process and resolve technological issues in emergencies. Third, in addition to the opinion of a few experts, we could exploit the value of collective knowledge and crowdsourcing data within structured consensus mechanisms.

\section{CLINICAL GUIDELINES AND PROTOCOLS}

With limited availability of evidence and the need for broad consensus meetings, relying only on eminences or slow bureaucratic orders is not the best choice. Instead, it is essential to prompt researchers and scientists to fully review "expert evidence" 4 by basing decisional frameworks on practical insights of stakeholders and frame recommendations as actionable points disseminated through multiple channels.

While a wave of changes in culture, technology, and practice is sweeping the globe, ${ }^{5}$ we have an unprecedented opportunity for optimizing the compromise between science and real-word decisions.

\section{About the Authors}

Department of Health and Social Policies, Autonomous Province of Trento, Trento, Italy (Dr Torri) and Department of Industrial Engineering - BIOtech Labs, University of Trento, Trento, Italy (Dr Nollo).

Correspondence and reprint requests to Emanuele Torri, Department of Health and Social Policies, Autonomous Province of Trento, Trento, Italy (e-mail: emanuele.torri@provincia.tn.it).

\section{Conflict of Interest}

The authors declare no conflict of interest.

\section{REFERENCES}

1. Farnoosh G, Zarei S, Hosseini Zijoud SS, et al. Is there a guarantee that the crisis of Covid-19 will not be repeated? Disaster Med Public Health Prep. 2020. doi: 10.1017/dmp.2020.83

2. Gagliano A, Villani PG, Có FM, et al. COVID-19 epidemic in middle province of northern Italy: impact, logistic \& strategy in the first line hospital. Disaster Med Public Health Prep. 2020. doi: 10.1017/dmp.2020.51

3. Anderson M, Mckee M, Mossialos E. Covid-19 exposes weaknesses in European response to outbreaks. BMJ. 2020;368: m1075. doi: 10.1136/bmj.m1075 
4. Schünemann HJ, Zhang Y, Oxman AD, et al. Distinguishing opinion from evidence in guidelines. BMJ. 2019;366:14606. doi: 10.1136/bmj.14606

5. Lichfield G. We're not going back to normal. Social distancing is here to stay for much more than a few weeks. It will upend our way of life, in some ways forever. MIT Technology Review; March 17, 2020. https://www. technologyreview.com/s/615370/coronavirus-pandemic-social-distancing18-months/?truid=a497ecb44646822921c70e7e051f7fla. Accessed April 21, 2020 . 